\title{
"Integrating China in the International Consortium for Personalized Medicine": The Coordination and Support Action to Foster Collaboration in Personalized Medicine Development between Europe and China
}

\author{
Alisha Morsella ${ }^{a} b \quad$ Chiara Cadeddu $^{b}$ Carolina Castagna ${ }^{b} \quad$ Ilda Hoxhaj ${ }^{b}$ \\ Michele Sassano $^{b}$ Chiuhui Mary Wang ${ }^{c}$ Lili Wang $^{d}$ Svetlana Klessova ${ }^{e, f}$ \\ Antonio Giulio de Belvis ${ }^{a}$ b Stefania Boccia ${ }^{b, g}$ Walter Ricciardi ${ }^{b}, h$ \\ ${ }^{a}$ Critical Pathway and Outcome Evaluation Unit, Fondazione Policlinico Universitario A. Gemelli IRCCS, Rome, Italy; \\ bSection of Hygiene, University Department of Life Sciences and Public Health, Università Cattolica del Sacro Cuore, \\ Rome, Italy; ' ${ }^{C}$ lobal Partnerships \& Projects Fondazione Telethon, Milan, Italy; ${ }^{d B G I}$ Research, Beijing Genomics \\ Institute BGI Group, Beijing, China; eUniversity Côte d'Azur, CNRS, GREDEG, Nice, France; ${ }^{\mathrm{f} G . A . C ., ~ I n n o v a t i o n ~}$ \\ Department., G.A.C. Group, Issy-les-Moulineaux, France; 9Department of Woman and Child Health and Public \\ Health-Public Health Area, Fondazione Policlinico Universitario A. Gemelli IRCCS, Rome, Italy; hIstituti Clinici \\ Scientifici Maugeri IRCCS, Pavia, Italy
}

\section{Keywords}

China $\cdot$ Genetic/genomic tools · Horizon $2020 \cdot$ Personalized medicine $\cdot$ Public health

\footnotetext{
Abstract

"Integrating China in the International Consortium for Personalized Medicine" (IC2PerMed) is a coordination and support action funded within the Horizon 2020 work program. Following the guidance of the International Consortium for Personalized Medicine (ICPerMed), the project's overarching aim is to align the European Union and China's research agendas in the field of personalized medicine (PM) to enable a swift development of PM approaches in the EU with strong leverage upon EU-Chinese collaboration. Living in the COVID-19 era, we are witnessing how the challenges imposed by the pandemic all around the globe have been acting as a catalyst for collaborations and knowledge sharing among national health systems worldwide. Given the strong inter-
}

est on behalf of both Europe and China in the advancement of PM approaches, now more than ever, a cross-border collaboration between the 2 powers can accelerate the effective translation of such innovation to healthcare systems, advance research, and ensure that such change follows the directions toward the path of sustainability. IC2PerMed developments will be led by European and Chinese experts equally assembled into 3 Working Groups: (1) people and organization, (2) innovation and market, and (3) research and clinical studies in PM. This complex and dynamic network of actions thrives on dialog, cooperation, and alignment of research at national and global levels; work in the direction taken by IC2PerMed shall pave the way toward the realization of PM's full potential, prevent it from becoming a burden for healthcare systems, and, rather, prove that it provides an essential and irreplaceable contribution to their effectiveness, efficiency, and sustainability.

(c) 2021 The Author(s).

Published by S. Karger AG, Basel karger@karger.com www.karger.com/phg

Karger $\frac{1}{\%}$

BOPEN ACCESS
(C) 2021 The Author(s)

Published by S. Karger AG, Basel

This is an Open Access article licensed under the Creative Commons Attribution-NonCommercial-4.0 International License (CC BY-NC) (http://www.karger.com/Services/OpenAccessLicense), applicable to the online version of the article only. Usage and distribution for commercial purposes requires written permission. 
"Integrating China in the International Consortium for Personalized Medicine" (IC2PerMed) is a coordination and support action (CSA) within the Horizon 2020 work program topic "Better health and care, economic growth, and sustainable health systems," under the work pillar "Health demographic, change, and wellbeing" [1]. Following the guidance of the International Consortium for Personalized Medicine (ICPerMed), the project's overarching aim is to align the European Union's (EU) and China's research agendas in the field of personalized medicine (PM), to enable a swift development of PM approaches in the EU with strong leverage upon EU-Chinese collaboration. IC2PerMed, funded under the H2020 grant agreement No. 874694 and also awaiting for the outcome of applications sent to the MOST, tackles the challenges of the topic HCO-012019 , with specific reference to the internationalization field.

Indeed, as called for by United Nation's Sustainable Development Goal 3, whose mission is to "ensure healthy lives and promoting wellbeing for all at all ages" [2], PM tailors disease prevention, diagnostics, and therapies to individual genetic profiles, improving outcomes, efficiency, safety, and treatment length. Recognizing such potential and supporting the best approaches to evolve health systems toward more efficient and sustainable ecosystems, the European Commission has notably set $\mathrm{PM}$ at the top of its research agenda supporting projects across the entire value chain, from research to service delivery.

Despite each member state maintaining its own responsibility in funding, provision and reimbursement systems, based either on public social insurance schemes or general taxation, the overall social healthcare model in European countries makes the continent an ideal setting to lead the way for a scale up of health system management, especially given efforts on behalf of the EU to align standards and national laws and regulations on the protection of people's health. Certainly, in a territory dominated by the principle of universal coverage, ensuring that the benefits of PM in the burden of NCDs are accessible by entire populations certainly does not come without its challenges: such new technologies and innovations call for advances at organizational, service, and system levels that are able to support the diffusion of new drugs, new digital solutions, and new reimbursement schemes [3].

Likewise, $\mathrm{PM}$ has been attracting massive interest in China which had included it in its 5-year research plan that specifically addressed genetics, biotechnology, cut-

IC2PerMed for Personalized Medicine in Europe and China ting-edge data collection, and analytical tools already in 2016 [4]. China is showing a strong commitment toward tackling the burden of NCDs and health service inequalities by allocating major investments toward multisectoral collaboration and innovation [5-7]. Specifically, much attention has been absorbed by the cancer burden and led to strategies for health education promotion, awareness raising, early diagnosis enablement, and treatment efficacy improvement, in an effort to balance out disproportionate economic development and inequitable access to care throughout the country. In fact, making up one-fifth of the world population and boasting an incredible diversity of geographical and cultural environments, China's wide spectrum of common and rare diseases, in different stages and with heterogeneous gender and geographical distributions, provides unique inputs in research toward a healthy world. Indeed, despite certainly complicating disease control mechanisms and management, the country offers the world diverse and valuable experiences [6] that constitute an ideal departure point for PM uptake when also considering how the holistic and individual specific philosophy of traditional Chinese medicine has always merged the macro- and the microenvironments of pathogenesis.

The outline of the 2 ecosystems proves how both are aiming toward the cooperation on innovation and promotion of a mutually beneficial, open, and equal science. Here, international backing between EU and China to address global challenges supports several joint projects between European and Chinese universities, research institutions, and companies as indicated by the Co-Funding Mechanism (CFM) renewed for the years 2018-2020. Thus, given the strong interest on behalf of both Europe and China in the advancement of PM approaches, a resistant and sustainable cross-border collaboration between the 2 powers can build a lever that is efficient in accelerating the effective translation of such innovation to healthcare systems, advance research, and ensure that such change follows the directions toward the path of sustainability.

Consequently, and as indicated by the EU's strategy and ICPerMed's Action Plan [8], the IC2PerMed project, intends to "provide those key solutions necessary to enable such collaboration by integrating relevant Chinese entities in the consortium and converge European and Chinese stakeholders toward a common approach in PM research, innovation, development, and implementation." To impact the EU's and China's strategic policy levels and their research and development activities, IC2PerMed bases 
its functioning on an active engagement of PM experts, policy makers, healthcare beneficiaries, patients, and societal stakeholders. For the transformation of clinical and biomedical research results into routine clinical practice to occur, a tight cooperation not only among researchers, clinicians, and patients but also between academic institutions and the industry has been deemed essential. Thus, an intersectoral approach has been chosen for this project, as a means to reach a continuous exchange of knowledge and innovative solutions [9]. The CSA has purposely assembled a total of 10 partners that can be considered leaders in the PM research field, with organizations deriving from Italy, Germany, Austria, Switzerland, France, and China, plus worldwide umbrella organizations such as the World Federation of Public Health Association, as a public health representative, and BBMRI-ERIC, as a European research infrastructure representative. The partners include research organizations - the Università Cattolica del Sacro Cuore (Rome, Italy), which is the project leader, and Tsinghua University (Beijing, China); two research healthcare organizations - Fondazione Policlinico A. Gemelli IRCCS (Rome, Italy) and the National Cancer Centre of the Chinese Academy of Medical Sciences (Bejing, China); one charitable foundation - Fondazione Telethon (Rome, Italy); and companies and networks such as the Beijing Genomics Institute (BGI) Life Science Research Institution, Steinbeis 2i (Stuttgart, Germany), and GAC Group (Sophia Antipolis, France). Supporting partners also include the Italian Ministry of Health, the European Alliance for Personalized Medicine, the European Public Health Association, Instruct-ERIC from Europe, the National Institute for Food and Drug Control, and the China National Health Development.

This project will be carried out in a 4 -year time frame, from January 2020 to December 2023. Initially, existing policies, programs, standards, and initiatives regarding the development and implementation of PM in Europe and China will be mapped, to distinguish opportunities for research collaborations and to pave the way for European and Chinese experts to exchange knowledge, know-how, and expertise by means of workshops and delegation visits. Developments will be, in fact, led by such teams of experts equally assembled into 3 Working Groups carrying out discussions aimed at delivering affordable yet high-quality personalized healthcare guided by the ICPerMed's Action Plan Priorities.

Specifically, WG1 "people and organization" focuses on matters of education and both citizens' and patients' engagement at microlevel (hospital and healthcare organization), mesolevel (integrated care and local level), and macrolevel (health system) ensuring their sustainability by including in experts' agenda also the update of academic curricula and methods to increase population health literacy and patient empowerment.

Second, WG2, "innovation and market," recognizes medical, genetic, and environmental data schemes as the inputs to generate innovative diagnostic methods, treatments, prognoses, and drug developments. Consequently, international alignment in these domains (from standards to market regulation and barriers) is of uttermost importance for collaboration and constitutes the aim of this WG. Focus will also be on health economic models, taking into consideration the specificities of pricing, insurance, and reimbursement models. In fact, experts will be involved in providing Health Technology Assessment guidelines for resource allocation and investment prioritization and in validating novel business models to bring innovations into the markets of health promotion, disease prevention, and treatment selection, so that PM can be made affordable at point of care, for all.

Last, WG3, "research and clinical studies in PM," includes topics such as classification, trials, and programs, aiming to build biobanking collaborations, defining standardization of approaches to translate basic to clinical research, and aligning the research funding priorities and procedures in the field of PM across the relevant funding agencies in EU and China.

Every step of this way will follow the path toward equity, sustainability, and value-addition in healthcare for China and the EU, to leverage the effectiveness of precision treatment and improve long-term societal health for all citizens. Last but not least, WG discussions will also include social and cultural aspects of both territories, particularly to understand barriers, obstacles, and drivers of stakeholder integration, deemed essential for the uptake of PM at local level.

The result of such synergies will first be converted into a roadmap leading toward the transferability and scaling up of international standards in PM, as the basis for interactions with ICPerMed and Chinese working agencies. Subsequently, a set of final recommendations issued by the experts, with feedback from funding agencies and policy makers, will be disseminated to encourage research collaborations and align priorities between Chinese programs and ICPerMed funders.

The outcome of the project aims to overcome traditional research funding schemes, to include communi- 
cation modules, outreach activities, and nonresearch cross-sectorial projects. Publicly accessible deliverables will include scoping papers and reports on health research and innovation priorities, on PM programs in Europe and China, and on barriers and opportunities for the collaboration, including one specific framework for Biobank Infrastructure alignment and one report on recommendations for international networks and policy outreach.

As it shows, the focus is on the entire health continuum, from research and policy implementation and from preventive medicine to palliative care, and the addressees are regulatory bodies, policy makers, healthcare providers, ethical, legal, and social experts, and patient/citizen organizations. Valuing the potential of PM in overcoming ineffective treatments improving outcomes, efficiency, safety, and treatment length is the prime driver of IC2PerMed's action plan, framed under the UN SDGs and the Horizon Call. During the years and as the project unfolds, IC2PerMed will build bridges and stimulate unceasing interactions with key organisms in both territories, for concrete developments to be placed within international contexts and under the ICPerMed umbrella's relevant initiatives and networks. Directly addressing SDG 3, the project's ultimate goal is to impact population health, building a system that maximizes empowerment and engagement of citizens from before they become patients, and identifying strategies that, faithful to the guiding principles of universal coverage, improve accessibility to quality services, affordability, and protect populations from financial hardship, turning PM into an opportunity for all.

Notably, as we navigate through the COVID-19 era, our population's health is being attacked on 2 fronts: one from SARS-CoV-2 and the other from the everlasting burden of NCDs. Thus, the scientific community is calling for what is being referred to as a "syndemic" approach [10] according to which the most effective strategy to fight both enemies at once is to jointly consider individual's biological factors and social determinants. Indeed, IC2PerMed aims to embed and implement its developments starting from a social, societal, and economic knowledge base to issue actionable recommendations in both healthcare systems for them to become more easily adaptable and responsive. Now more than ever, we are witnessing how the challenges imposed all around the globe by the pandemic have been acting as a catalyst for collaborations and knowledge sharing among national health systems worldwide and demonstrated that clinical practice and disease management can gain substantial benefits from cross-border exchanges of experiences and management models, despite differences in population or country size, mortality or morbidity rates, or geographical distance [11]. This complex and dynamic network of actions thrives on dialog, cooperation, and alignment of research at national and global levels; work in the direction taken by IC2PerMed shall pave the way toward the realization of PM's full potential, prevent it from becoming a burden for healthcare systems, and, rather, prove that it provides an essential and irreplaceable contribution to their effectiveness, efficiency, and sustainability [12].

\section{Conflict of Interest Statement}

The authors have no conflicts of interest to declare.

\section{Funding Sources}

This project has received funding from the European Union's Horizon 2020 research and innovation program under grant agreement No. 874694 .

\section{Author Contributions}

Alisha Morsella, Chiara Cadeddu, Carolina Castagna, Ilda Hoxhaj, and Michele Sassano drafted, reviewed, and revised the manuscript. Chiuhui Mary Wang, Svetlana Klessova, and Lili Wang contributed to the drafting and revision of the manuscript. Walter Ricciardi, Stefania Boccia, and Antonio Giulio de Belvis critically reviewed the manuscript providing important intellectual content. All authors approved the final manuscript as submitted and agree to be accountable for all aspects of the work.

References

Public Health Genomics 2021;24:310-314 313
1 European Commission. Health, Demographic Change and Wellbeing [Internet]. Available from: https://ec.europa.eu/programmes/ horizon2020/en/h2020-section/health-demographic-change-and-wellbeing.

2 United Nations. Goal 3: Ensure healthy lives and promote well-being for all at all ages [Internet]. Available from: https://www.un.org/ sustainabledevelopment/health/.

3 Westerling R, Westin M, McKee M, Hoffmann R, Plug I, Rey G, et al. The timing of introduction of pharmaceutical innovations in seven European countries. J Eval Clin Pract. 2014;20(4):301-10.

4 13th five years plan for Economic and social development of the People's Republic of China (2016-2020), translation. Available from: http: //en.ndrc.gov.cn/newsrelease/201612/ P020161207645765233498.pdf. 
5 Ministry of Foreign Affairs of the People's Republic of China. The China's progress report on implementation of the 2030 Agenda for sustainable development (2019). 2019. Available from: https://www.fmprc.gov.cn/mfa eng/topics_665678/2030 kcxfzyc/ P020190924780823323749.pdf.

6 Kong L-Z. China's medium-to-long term plan for the prevention and treatment of chronic diseases (2017-2025) under the healthy China initiative. Chronic Dis Transl Med. 2017;3(3):135-7.
7 Healthy China Action Plan 2019-2030. 2020. Available from: http://en.nhc.gov.cn/ HealthyChinaActionPlan.html.

8 The ICPerMed Action Plan (2017). Available from: https://www.icpermed.eu/media/content/ICPerMed_Actionplan_2017_web.pdf.

9 The ICPerMed Vision for 2030: how can personalised approaches pave the way to nextgeneration medicine? Available from: https:// www.icpermed.eu/media/content/Vision Paper_2019.pdf.
10 Horton R. Offline: COVID-19 is not a pandemic. Lancet. 2020 Sep 26;396(10255):874.

11 Chen J, Lu H, Melino G, Boccia S, Piacentini M, Ricciardi W, et al. COVID-19 infection: the China and Italy perspectives. Cell Death Dis. 2020;11(6):438. Published 2020 Jun 8.

12 Ricciardi W, Boccia S. New challenges of public health: bringing the future of pesonalised healthcare into focus. Eur J Public Health. 2017 Oct 1;27(Suppl 4):36-9. 